\title{
UMA ABORDAGEM EXATA E META-HEURÍSTICAS PARA O P-CABLE TRENCH PROBLEM WITH COVERING
}

\author{
Ítalo Fernandes Gonçalves e Alexandre Ribeiro \\ Pontifícia Universidade Católica de Goiás, Setor Leste Universitário, Goiânia - GO, 74175-120, Brasil
}

\begin{abstract}
RESUMO
Neste trabalho é abordado o p-cable trench problem with covering (pCTPC), um problema de otimização combinatória NP-completo, recentemente introduzido na literatura. Seu objetivo é minimizar o custo total da instalação de servidores primários e servidores secundários a fim de satisfazer toda demanda de usuários de uma região. Os lugares onde, exclusivamente, podem ser instalados os servidores são os nós da rede, que coincidem com as localidades dos usuários. Tanto servidores primários quanto secundários distribuem os dados dentro de seu raio de cobertura, porém somente os primários são fornecedores de dados. Dessa forma é necessário que cada servidor secundário seja conectado através de uma conexão dedicada a um primário, evitando o compartilhamento da capacidade de transmissão de dados pelos cabos. Neste trabalho é apresentado uma adaptação da formulação linear inteira do pCTPC, baseada em trabalhos da literatura e propostas três abordagens, sendo a primeira uma abordagem heurística baseada no Biased random-key genetic algorithm (BRKGA) e a segunda baseada no Variable neighborhood search (VNS) e a terceira uma solução exata com o resolvedor de programação linear inteira GUROBI. O resolvedor encontrou o ótimo para todas instâncias e foi utilizado para comparação e validação das abordagens heurísticas. As propostas demonstram ser competitivas e promissoras.
\end{abstract}

\section{PALAVRAS-CHAVE}

Meta-heurísticas, Otimização combinatória, Programação linear inteira, Posicionamento de Servidores com Cobertura

\section{INTRODUÇÃO}

Posicionamento de servidores com raio de cobertura é uma situação que ocorre em empresas de distribuição de dados. É preciso garantir que todos clientes de uma rede sejam atendidos por um serviço (serviço de internet, por exemplo). Geralmente são posicionados servidores primários, que são fornecedores do serviço,

e servidores secundários, que apenas replicam os dados recebidos, isto é, dependem dos servidores primários para seu funcionamento. Decidir onde serão instalados cada servidor pode ser uma tarefa complexa que demanda tempo e com gastos altamente significantes.

Esse tipo de situação pode ser comparada ao $p$-Cable Trench Problem with Covering (pCTPC). No pCTPC, como definido em Marianov et al. (2015), tanto servidores primários como secundários possuem um raio de cobertura $r$ e atendem os clientes finais. O problema é definido sobre um conjunto de pontos de demanda, locais estes onde, exclusivamente, podem ser instalados os servidores. Cada servidor secundário deve ser conectado através de uma conexão dedicada, via cabo, a um servidor primário. Os cabos devem ser colocados dentro de valas que estarão posicionadas entre os pontos de demanda. Nem sempre entre dois pontos de demanda é possível posicionar uma vala, considerando problemas físicos reais como barreiras naturais ou artificiais. Existe um custo de construção das valas e um custo por unidade de comprimento dos cabos.

O objetivo é minimizar o custo de instalação das valas, bem como o custo dos cabos utilizados, garantindo que todos os pontos de demanda sejam cobertos por pelo menos um servidor (primário ou secundário) usando exatamente $p$ servidores primários e um número arbitrário de servidores secundários (Marianov et al. 2015).

Considere como exemplo uma empresa que deseja fornecer o serviço de internet sem fio para seus clientes. Os servidores primários serão os roteadores e os servidores secundários serão as antenas Wi-Fi. A empresa se encontra em uma região onde já existe um rede telefônica legada, isto é, apenas serviços de voz e dados são oferecidos. Os únicos lugares nos quais podem ser instalados servidores são os pontos já presentes na rede. A Figura 0 apresenta o exemplo de uma rede de clientes dessa empresa.

Essa empresa decide alocar exatamente dois roteadores primários, isto é, $p=2$, e a fim de cobrir toda a rede, posiciona cinco antenas Wi-Fi. A configuração descrita é apresentada na Figura 1(b). A Figura 1(c) ilustra os servidores com seus raios de cobertura, em azul o raio de cobertura de servidores primários e em vermelho o raio de cobertura de servidores secundários. Já na 1(d) é apresenta uma configuração válida para a conexão dos cabos. 


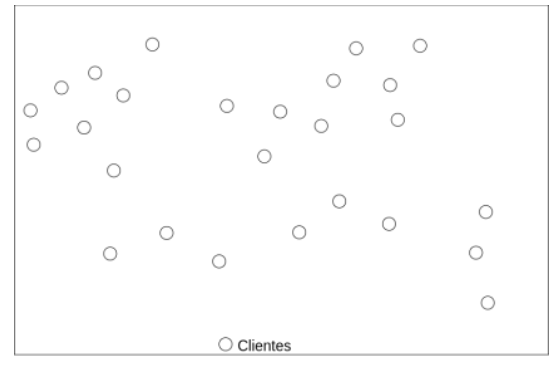

(a) Rede antes sem cobertura Wi-Fi.

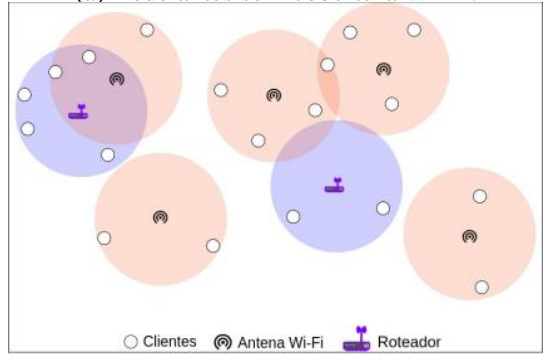

(c) Servidores com raios de cobertura.

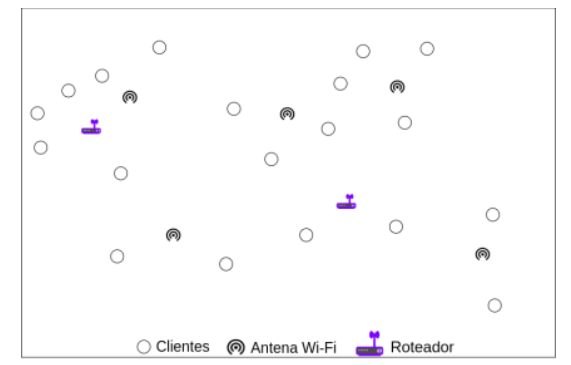

(b) Rede com servidores posicionados.

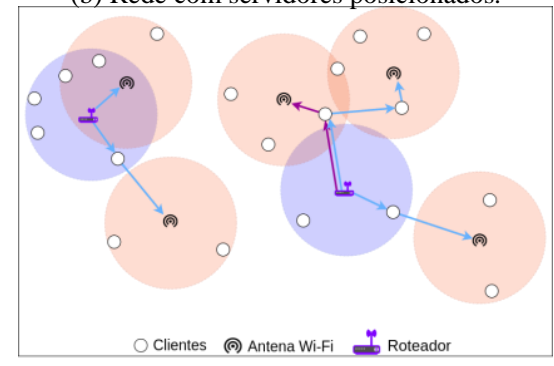

(d) Solução factível.

Figura 1. Aplicação do pCTPC. Adaptada de Calik et al. (2016)

O pCTPC é uma variação do Cable Trench Problem (CTP), em que todos os nós são considerados como servidores e devem estar conectados, através de uma conexão dedicada, a um único nó primário da rede, sendo os demais secundários (Vasko et al. 2002). Dentre as aplicações destes problemas e suas variantes pode-se citar o planejamento de redes cabeadas e sem fio e reconstrução de vascularização em imagens de tomografia computadorizada (Vasko et al. 2015).

Trata-se de um problema pelo menos NP-completo (Marianov et al. 2015), sendo uma generalização do CTP, que foi provado ser NP-completo por Vasko et al. (2002). Assim, diante do fato de, provavelmente, não ser praticável obter uma solução ótima em tempo polinomial para o pCTPC, abre-se espaço para a utilização de abordagens heurísticas para tratar o problema.

Neste trabalho é apresentada uma formulação em programação linear inteira adaptada de outros trabalhos da literatura (Marianov et al. 2015, Calik et al. 2016) para o pCTPC e são propostas três abordagens para tratá-lo, sendo a solução exata utilizando o resolvedor de programação linear inteira GUROBI e a duas heurísticas, uma delas baseadas no Biased random-key genetic algorithm e outra no Variable neighborhood search.

\section{O PROBLEMA: P-CABLE TRENCH PROBLEM WITH COVERING}

O pCTPC é um problema de otimização combinatória que possui o objetivo de selecionar $p$ nós primários e um número arbitrário de nós secundários, sendo que cada nó primário e cada nó secundário distribuem o sinal até uma distância $r$ de si, em seu raio de cobertura, cujo objetivo é cobrir todos os nós com custo mínimo, levando em consideração o custo da conexão dedicada entre nós primários e secundários, via cabo, e o custo de construção das valas, por onde os cabos devem serem passados.

\subsection{Definição Formal}

Considere o grafo direcionado $G=(V, A)$, onde $V$ é o conjunto de vértices e $A$ é o conjunto de arestas. Considere também os conjuntos $J, S$ e $I$, respectivamente: conjunto de clientes, servidores primários e servidores secundários. Pode-se dizer que $V=J$, isto é, todo vértice é um cliente e que $S \subseteq I$, assim como $I \subseteq J$, indicando que existe um subconjunto dentre todos clientes que também são servidores secundários e que dentro deste conjunto existe um subconjunto em que todos são servidores primários. 
Formalmente o problema pCTPC pode ser definido da seguinte maneira: dado o grafo $G=(V, A)$, encontrar os subconjuntos $S$ e $I$, respectivamente, de exatamente $p$ servidores primários e um número arbitrário para servidores secundários, a fim de cobrir todos os vértices do grafo, tal que $\forall j \in J$ deve estar dentro do raio de cobertura fornecido por um servidor secundário $i \in I$, ou servidor primário $s \in S$, minimizando, assim o custo de distribuição do serviço. Custo este que envolve a construção de valas e o custo das conexões dedicadas, via cabo, entre servidores primários e secundários. O custo dos servidores não foi levado em consideração, pois considera-se que o número de servidores é constante na maioria dos casos para cobrir toda a rede, assim como afirmado em Marianov et al. (2015).

\subsection{Formulação Matemática}

Nos trabalhos de Marianov et al. (2015) e Calik et al. (2016), o pCTPC foi modelado como um problema de programação linear inteira. Na formulação foi inserido o vértice $0 \mathrm{em} V$, assim como todas arestas, $(0, j)$ $\forall j \in V$, inseridas em $A$. Denota-se então o grafo $G_{0}=\left(V_{0}, A_{0}\right)$ com $V_{0}=V \cup\{0\}$ e $A_{0}=A \cup$ $\{(0, j) \mid j \in V\}$. O nó adicionado, facilita a imposição de algumas restrições como pode ser observado mais adiante. Este trabalho apresenta a formulação nas equações (1) - (10), com uma adaptação na restrição de equilíbrio de fluxo.

Cada solução é representada como uma árvore enraizada neste novo vértice com exatamente $p$ filhos. São variáveis de decisão binaria $x_{u v} \in\{0,1\}, \forall(u, v) \in A_{0}$, que será igual a um quando a aresta $(u, v)$ pertence a solução, e cada variável $g_{u v}^{i} \in\{0,1\}, \forall i \in V, \forall(u, v) \in A_{0}$, será igual a um se, e somente se, a aresta $(u, v)$ está no caminho da raiz, ou seja, do vértice 0 , até o vértice $i$, no caso deste vértice ser secundário. A variável $y_{i} \in\{0,1\}, \forall i \in I$, indica se um servidor secundário está instalado em $i$, se, e somente se, for igual a um. Sejam os custos da vala a ser construída na aresta $(u, v)$, dado por $f_{u v}, \forall(u, v) \in A$ e o custo do cabo $c_{u, v}, \forall(u, v) \in$ $A$. Seja também a variável $z_{i j} \in\{0,1\}, \forall i \in V, \forall j \in V$, que será igual a um se, e somente se, dist $t_{i j} \leq r$, sendo $j$ um possível cliente que é alcançável pelo possível servidor em $i$. Por fim, dado o vértice $u$, denomina-se, $\gamma^{+}(u)=\left\{(u, v) \in A_{0}\right\}$, o conjunto de todas as arestas que partem do vértice $u$ e $\gamma^{-}(u)=\left\{(v, u) \in A_{0}\right\}$ como todo o conjunto de arestas incidentes em $u$. minimizar:

sujeito a:

$$
\sum_{(u, v) \in A} f_{u v} x_{u v}+\sum_{i \in V} \sum_{(u, v) \in A} c_{u v} g_{u v}^{i}
$$

$$
\sum_{(v, u) \in \gamma^{+}(v)} g_{v u}^{i}-\sum_{(u, v) \in \gamma^{-}(v)} g_{u v}^{i}=\left\{\begin{array}{ll}
y_{i}, & \text { se } v=0 \\
-y_{i}, & \text { se } v=i \\
0, & \text { caso contrário }
\end{array} \quad \forall i \in V, \forall v \in V_{0}\right.
$$

$g_{u v}^{i}-x_{u v} \leq 0$

$\sum_{(u, v) \in A_{0}} x_{u v} \leq$
$\sum_{v \in V}^{v \in V} x_{0 v}=p$
$\sum_{i \in V} z_{i j} y_{i} \geq 1$

$$
\begin{aligned}
& \forall i \in V, \forall(u, v) \in A_{0} \\
& \forall v \in V \\
& \forall j \in V \\
& \forall i \in V, \forall(u, v) \in A_{0} \\
& \forall(u, v) \in A_{0} \\
& \forall i \in V, \forall j \in V \\
& \forall i \in V
\end{aligned}
$$$$
g_{u v}^{i} \in\{0,1\}
$$$$
x_{u v} \in\{0,1\}
$$$$
z_{i j} \in\{0,1\}
$$$$
y_{i} \in\{0,1\}
$$

O objetivo da função (1) é minimizar a soma do custo de construção das valas e do custo do cabo. As equações em (2) garantem que haverá conexão física de um servidor primário a um servidor secundário. A restrição (3) certifica que haverá cabos somente em arestas onde serão construídas as valas. As restrições (4) e (5) garantem que a solução seja uma arborescência em $G_{0}$, enraizada na raiz artificial 0 com exatamente $p$ filhos. As desigualdades (6) afirmam que cada demanda deve ser atribuído a um servidor secundário. As restrições de (7) - (10) certifica que cada variável esteja dentro de seu domínio. 


\section{TRABALHOS RELACIONADOS}

O cable trench problem (CTP), já citado, proposto por Vasko et al. (2002) que o resolveu usando um modelo de programação linear inteira. Jeng, Kim e Watada (2007) propruseram uma heurística baseada no algoritmo molecular. Anos depois, Marianov et al. (2012) consideram a demanda igual a 1 em todos os nós da rede e determinam $p$ instalações, definindo essa variação como p-cable trench problem (pCTP) e propõem um modelo de programação linear inteira, resolvendo-o com heurísticas baseadas no relaxamento lagrangiano.

Introduzido por Marianov et al. (2015), o pCTPC foi proposto juntamente com seu modelo de otimização linear baseado no fluxo de multi-comodidade. No trabalho, foram propostas 3 soluções para o problema, sendo um relaxamento linear seguido de ramificação e limite quando necessário, e duas heurísticas baseadas no relaxamento lagrangiano para determinar soluções viáveis. As abordagens adotadas foram testadas em instâncias de 100 e 200 nós e usada para resolver um caso real que busca determinar a melhor localização para um número adequado de antenas Wi-Fi e $p$ roteadores, a fim de fornecer serviço de internet a uma área turística de a cidade de Viña del Mar no Chile.

Calik et al. (2016) propôs o Capacitated p-Cable Trench Problem with Covering (CpCTPC), uma variação do pCTPC com restrição do número de conexões entre servidores primários e secundários. É apresentada uma estrutura algorítmica baseada na decomposição de Benders para resolver o CpCTPC, além de duas formulações de programação inteira baseadas em fluxo serem apresentadas. $\mathrm{O}$ algoritmo proposto conta com um procedimento de estabilização para acelerar a convergência do loop de corte e deriva soluções primárias de alta qualidade com uma heurística primária. Um ponto levantado é a versatilidade do algoritmo que pode ser aplicado a maioria das variantes do CTP.

\section{ABORDAGENS ADOTADAS}

\subsection{Resolvedor de Programação Linear Inteira}

A formulação apresentada foi aplicada a um resolvedor comercial de programação linear inteira. Optou-se por utilizar a framework GUROBI na versão 9.0 (Gurobi Optimization 2020), por ser um dos resolvedores mais bem avaliados em testes de performance (Meindl \& Templ 2013, Souza et al.2017).

O GUROBI reduz as dimensões do problema, excluindo variáveis e restrições redundantes. Ele executa um método de busca baseado no algoritmo Branch-and-Bound, além de outras estratégias como o paralelismo e a detecção de simetria (Gurobi Optimization 2020).

\subsection{Biased Random-Key Genetic Algorithm}

O Biased random-key genetic algorithm (BRKGA) é uma meta-heurística evolutiva proposta por Gonçalves \& Resende (2011). Ela é inspirada na seleção natural de Darwin, como no genetic algorithm (GA) (Goldbarg 2016), em que sua principal característica é ser altamente elitista, perpetuando sempre os melhores cromossomos da população e atribuindo maior probabilidade para que as melhores características sejam propagadas durante o processo evolutivo.

Os cromossomos geralmente são representados como um vetor de chaves aleatórias de tamanho fixo $n$, em que uma chave pertence ao intervalo contínuo [0,1) (Gonçalves \& Resende 2011). Para se obter uma solução é necessário decodificar o cromossomo, através de um processo determinístico. $\mathrm{O}$ algoritmo é inicializado com uma população de $p$ indivíduos, em que cada indivíduo vetor de tamanho $n$, onde cada posição contém uma chave aleatória. A população então é classificada a cada geração e dividida em dois grupos: o grupo elite que consiste nos $p_{e}$ melhores indivíduos da população, de acordo com o valor de sua solução, sendo $p_{e}<\frac{p}{2}$ e o segundo grupo não elite, com indivíduos restantes, consistindo de $p-p_{e}$ indivíduos (Gonçalves \& Resende 2011).

Os indivíduos da próxima geração consistem no grupo elite, mais $p_{m}$ vetores mutantes gerados aleatoriamente, onde $p m<\frac{p}{2}$ e o restante $\left(p-p_{e}-p_{m}\right)$ são gerados através da etapa de cruzamento. O cruzamento é uma etapa importante do BRKGA, por tratar da característica principal de chaves viciadas. É escolhido um indivíduo $a$ do grupo elite e um indivíduo $b$ do grupo não elite, tais indivíduos serão os pais do 
indivíduo $c$ gerado nesta operação. A i-ésima chave de $c$ vai receber a chave do pai $a$ com probabilidade $p_{a}$ ou irá receber a chave do pai $b$ com probabilidade $1-p_{a}$, sendo que $p_{a}>\frac{1}{2}$.

\subsubsection{Codificação da Solução para o pCTPC}

Na proposta realizada neste trabalho, cada cromossomo foi codificado em dois vetores de chaves aleatórias. $\mathrm{O}$ primeiro vetor $V_{A}$ representa todas arestas do grafo e possui tamanho $|A|$. O segundo vetor $V_{V}$ representa todos os vértices do grafo e possui tamanho $|V|$.

\subsubsection{Decodificação para o pCTPC}

O passo inicial do decodificador é especificar os servidores primários e secundários. Para isso, é associada uma chave de $V_{V}$ para cada vértice e, então, os vértices são ordenados de maneira crescente pela chave. Os $p$ primeiros vértices da sequência serão servidores primários e os seguintes serão secundários enquanto houverem vértices descobertos. Lembrando que se um vértice é servidor, então ele cobre todos vértices a uma distância $r$ de si.

O passo seguinte é a definição das valas, para isso é atribuída uma aresta para cada chave de $V_{A}$. Para obter uma solução viável, as arestas são ordenadas de maneira crescente pela chave e iteradas nessa ordem para construir uma árvore geradora que define as valas utilizadas (caminhos), de maneira similar ao algoritmo de Kruskal (Halim \& Halim 2013), em que adiciona-se uma aresta à solução, caso ela não forme um ciclo. Os $p$ servidores primários são inseridos inicialmente conectados à raiz, nó artificial 0 .

Da forma realizada é possível que vértices folhas, que não são servidores, estejam conectados à árvore. Esses são nós indesejáveis para a solução, então é retirado cada um desses vértices utilizando uma aplicação do Breadth-First Search (BFS). Todos os vértices que são folhas e não são servidores são inseridos na fila inicial do BFS. Ao processar cada vértice da fila, desconecta-se este de seu vizinho e então é verificado se o vizinho agora é folha e se não é um servidor. Caso verdadeiro, este será inserido na fila para ser processado, até que não haja mais vértices com essas características, garantindo assim que a solução não terá arestas desnecessárias.

No passo final de decodificação é calculado o custo da solução através do algoritmo BFS, aplicado inicialmente no nó raiz (nó 0 ). Na fila do BFS, é inserido um par formado por índice que representa o vértice e soma dos cabos até este vértice a partir da raiz. Assim, quando alcançado um vértice secundário, é somado o custo de conexão (do nó 0 ao atual servidor secundário) ao custo total, assim como o custo de toda aresta visitada, isto é, o custo das valas, é inserido no custo total.

\subsection{Variable Neighborhood Search}

De acordo Mladenovic (2003), o Variable neighborhood search (VNS) é uma heurística que tem como principal característica a exploração de vizinhanças distantes da solução atual, e só muda para uma próxima vizinhança se houver uma melhoria. O método de busca local é aplicado repetidas vezes, na tentativa de se obter os ótimos locais da vizinhança atual.

O VNS utiliza de estruturas de vizinhança, as quais são utilizadas na etapa de busca local para encontrar as soluções vizinhas melhores que a solução inicial fornecida no início. O VNS possui como parâmetro de entrada o número de iterações e a quantidade de buscas a serem realizadas em uma vizinhança seguidas de melhorias.

\subsubsection{Representação da solução e solução inicial}

A solução foi codificada armazenando um vetor de vértices e um vetor de arestas, a fim de passar pelo mesmo processo de decodificação no BRKGA, sem a necessidade de mapeamento. A solução inicial consistiu na construção de uma árvore geradora mínima baseado no valor do cabo e no valor da vala com uma sequência randômica de vértices. A escolha da solução inicial se deu pelo fato de encontrar resultados que são intermediários e que aceleram o processo de otimização.

\subsubsection{Busca Local}

A busca local utilizada baseia-se na abordagem 2-opt (Helsgaun 2009). São sorteados aleatoriamente 2 posições e trocados seus valores ou invertida toda sequência entre essas duas posições. A busca local para o VNS, foi aplicada a estratégia 2-opt 4 vezes: aplicando-a em ambos vetores de vértices e arestas, trocando somente o conteúdo das posições e em outra aplicação com a inversão do intervalo entre as posições, adotando 
também a estratégia somente ao vetor de vértices, com uma troca tendenciosa, em que uma das posições sorteadas é necessariamente um vértice que aloca um servidor primário, trocando somente o conteúdo, e por fim o uso da estratégia somente no vetor de arestas, com troca do conteúdo das posições sorteadas, sem inversão. A cada execução da estratégia, a solução atual será atualizada se houver melhoria da solução atual.

\section{EXPERIMENTOS COMPUTACIONAIS}

As heurísticas foram implementadas na linguagem de programação Python utilizando utilizando o interpretador PyPy 7.3 (PyPy3.6). Para a formulação de programação linear inteira foi utilizada a API de Python do GUROBI na versão 9.0. Os testes foram processados em uma máquina de 16GB de memoria RAM, processador Intel Core i7-8565u, sobre o sistema operacional Ubuntu 19.10 (64 bits). Utilizou-se o gap como métrica de comparação entre abordagens. O gap é a distância em porcentagem de uma solução a outra solução de referência.

\subsection{Elaboração das Instâncias}

Foi gerado um conjunto de instâncias de benchmark baseadas no uncapacitated p-median problem disponíveis em Beasley (1990), nas instâncias de pmed01 a pmed10, seguindo um processo similar a Marianov et al. (2015). Nesse processo, em cada instância é descrito um grafo e o custo de suas arestas, que foram fixados como o custo da construção das valas. Foram retiradas as arestas duplicadas e calculado o caminho mínimo entre todos os pares $i$ e $j$, dado por SCij, através do algoritmo de floyd-warshall (Halim \& Halim 2013), atribuindo o valor do cabo com uma distribuição uniforme.

\subsection{Definição dos Parâmetros}

Foi realizado um breve estudo variando-se alguns parâmetros do BRKGA em algumas instâncias de teste. $p_{a}$ foi variada de 0.55 a 0.8 , assim como $p_{m}$ e $p_{e}$ variados de 0.1 a 0.35 , sendo que $p_{m}$ e $p_{e}$ são multiplicados pelo tamanho da população e arredondados para o inteiro mais próximo. A população foi fixada em 120 indivíduos. Com base nas experimentações realizadas e principalmente com valores recomendados por Gonçalves \& Resende (2011), foram definidas: $p_{a}=0.645, p_{m}=0.17$ e $p_{e}=0.28$. Para o problema, utilizou-se $r=\{6,7,8\}$ e $p$ igual a $5 \%$ de $|V|$, para todas instâncias, assim como realizado no trabalho de Marianov et al. (2015) para fim de comparação.

\section{RESULTADOS}

Devido ao fato do BRKGA trabalhar com pequenas melhorias ao decorrer do tempo e das iterações, foi determinado limitar o tempo de execução em 20 minutos. Para o VNS foi escolhido um critério de parada diferente, se atingido $L I M$ iterações sem melhorias, a execução do algoritmo é encerrada, onde $L I M=70 *$ $|V|$. O tempo de execução do GUROBI foi limitado em 1 hora, e devido ao seu bom desempenho foi utilizado como referência para cálculo do gap das abordagens heurísticas.

$\mathrm{Na}$ tabela 1 e 2 são apresentado os resultados das abordagens propostas neste trabalho, para resolver o pCTPC. A tabela 1 apresenta as instâncias de 100 vértices e 200 arestas e a tabela 2 as instâncias de 200 vértices e 800 arestas. A coluna $I$ identificando a instância, $r$ o raio de cobertura considerado, $T(s)$ o tempo em segundo que a abordagem gastou para executar a instância e Resultado o resultado alcançado. O gap da abordagem com o GUROBI é o gap reportado na saída de sua execução. Os demais gaps são calculados com base no resultado obtido por essa abordagem. 
Tabela 1. Resultado para as instâncias de 1 a 5, com 100 vértices

\begin{tabular}{|c|c|c|c|c|c|c|c|c|c|c|}
\hline \multirow{2}{*}{ I } & \multirow{2}{*}{$\mathbf{r}$} & \multicolumn{3}{|c|}{ GUROBI } & \multicolumn{3}{|c|}{ VNS } & \multicolumn{3}{|c|}{ BRKGA } \\
\hline & & $\mathbf{T}(\mathbf{s})$ & Resultado & Gap & $\mathbf{T}(\mathbf{s})$ & Resultado & Gap & $\mathbf{T}(\mathbf{s})$ & Resultado & Gap \\
\hline \multirow{3}{*}{1} & 6 & 17 & 5018 & $0 \%$ & 87 & 5019 & $0,02 \%$ & 1200 & 5100 & $1,61 \%$ \\
\hline & 7 & 25 & 5018 & $0 \%$ & 58 & 5070 & $1,03 \%$ & 1200 & 5106 & $1,72 \%$ \\
\hline & 8 & 51 & 4973 & $0 \%$ & 95 & 5012 & $0,78 \%$ & 1200 & 5028 & $1,09 \%$ \\
\hline \multirow{3}{*}{2} & 6 & 19 & 4566 & $0 \%$ & 93 & 4611 & $0,98 \%$ & 1200 & 4618 & $1,13 \%$ \\
\hline & 7 & 25 & 4496 & $0 \%$ & 86 & 4594 & $2,13 \%$ & 1200 & 4528 & $0,71 \%$ \\
\hline & 8 & 34 & 4485 & $0 \%$ & 64 & 4585 & $2,18 \%$ & 1200 & 4535 & $1,10 \%$ \\
\hline \multirow[b]{3}{*}{3} & 6 & 6 & 4987 & $0 \%$ & 91 & 5136 & $2,90 \%$ & 1200 & 5173 & $3,60 \%$ \\
\hline & 7 & 7 & 4975 & $0 \%$ & 93 & 5134 & $3,10 \%$ & 1200 & 5039 & $1,27 \%$ \\
\hline & 8 & 6 & 4946 & $0 \%$ & 46 & 5050 & $2,06 \%$ & 1200 & 5010 & $1,28 \%$ \\
\hline \multirow{3}{*}{4} & 6 & 4 & 5260 & $0 \%$ & 93 & 5463 & $3,72 \%$ & 1200 & 5264 & $0,08 \%$ \\
\hline & 7 & 4 & 5255 & $0 \%$ & 57 & 5342 & $1,63 \%$ & 1200 & 5382 & $2,36 \%$ \\
\hline & 8 & 14 & 5248 & $0 \%$ & 97 & 5375 & $2,36 \%$ & 1200 & 5281 & $0,62 \%$ \\
\hline \multirow{3}{*}{5} & 6 & 37 & 3963 & $0 \%$ & 89 & 4005 & $1,05 \%$ & 1200 & 4019 & $1,39 \%$ \\
\hline & 7 & 34 & 3935 & $0 \%$ & 62 & 3944 & $0,23 \%$ & 1200 & 4023 & $2,19 \%$ \\
\hline & 8 & 50 & 3913 & $0 \%$ & 97 & 4004 & $2,27 \%$ & 1200 & 3974 & $1,53 \%$ \\
\hline \multicolumn{2}{|c|}{ Média } & 22,2 & 4735,87 & $0 \%$ & 81 & 4822,93 & $1,76 \%$ & 1200 & 4805,33 & $1,45 \%$ \\
\hline
\end{tabular}

Tabela 2. Resultado para as instâncias de 6 a 10, com 200 vértices

\begin{tabular}{|c|c|c|c|c|c|c|c|c|c|c|}
\hline \multirow{2}{*}{$\mathbf{I}$} & \multirow{2}{*}{$\mathbf{r}$} & \multicolumn{3}{|c|}{ GUROBI } & \multicolumn{3}{|c|}{ VNS } & \multicolumn{3}{|c|}{ BRKGA } \\
\hline & & T(s) & Resultado & Gap & $\mathrm{T}(\mathrm{s})$ & Resultado & Gap & $\mathbf{T}(\mathrm{s})$ & Resultado & Gap \\
\hline \multirow{3}{*}{6} & 6 & 811 & 4502 & $0 \%$ & 801 & 4627 & $2,70 \%$ & 1200 & 5430 & $17,09 \%$ \\
\hline & 7 & 752 & 4437 & $0 \%$ & 1260 & 4555 & $2,59 \%$ & 1200 & 5004 & $11,33 \%$ \\
\hline & 8 & 1139 & 4403 & $0 \%$ & 1193 & 4485 & $1,83 \%$ & 1200 & 5371 & $18,02 \%$ \\
\hline \multirow{3}{*}{7} & 6 & 176 & 4719 & $0 \%$ & 1304 & 4804 & $1,77 \%$ & 1200 & 5638 & $16,30 \%$ \\
\hline & 7 & 163 & 4637 & $0 \%$ & 1113 & 4755 & $2,48 \%$ & 1200 & 5460 & $15,07 \%$ \\
\hline & 8 & 168 & 4583 & $0 \%$ & 1073 & 4676 & $1,99 \%$ & 1200 & 5512 & $16,85 \%$ \\
\hline \multirow{3}{*}{8} & 6 & 160 & 4966 & $0 \%$ & 1174 & 5078 & $2,21 \%$ & 1200 & 5885 & $15,62 \%$ \\
\hline & 7 & 124 & 4850 & $0 \%$ & 1474 & 4962 & $2,26 \%$ & 1200 & 5561 & $12,79 \%$ \\
\hline & 8 & 235 & 4809 & $0 \%$ & 1428 & 4944 & $2,73 \%$ & 1200 & 5659 & $15,02 \%$ \\
\hline \multirow{3}{*}{9} & 6 & 707 & 4962 & $0 \%$ & 1296 & 5117 & $3,03 \%$ & 1200 & 5678 & $12,61 \%$ \\
\hline & 7 & 374 & 4838 & $0 \%$ & 1088 & 4903 & $1,33 \%$ & 1200 & 5920 & $18,28 \%$ \\
\hline & 8 & 1258 & 4710 & $0 \%$ & 977 & 4805 & $1,98 \%$ & 1200 & 5457 & $13,69 \%$ \\
\hline \multirow{3}{*}{10} & 6 & 415 & 3728 & $0 \%$ & 1150 & 3850 & $3,17 \%$ & 1200 & 4485 & $16,88 \%$ \\
\hline & 7 & 731 & 3635 & $0 \%$ & 855 & 3727 & $2,47 \%$ & 1200 & 4430 & $17,95 \%$ \\
\hline & 8 & 1270 & 3469 & $0 \%$ & 919 & 3582 & $3,15 \%$ & 1200 & 4288 & $19,10 \%$ \\
\hline \multicolumn{2}{|c|}{ Média } & 565,53 & 4483,20 & $0 \%$ & 1.140 & 4591,33 & $2,38 \%$ & 1200 & 5318,53 & $15,77 \%$ \\
\hline
\end{tabular}

A tabela 3 apresenta os resultados para instâncias de mesmo tamanho que Marianov et al. (2015) utilizou em seu trabalho, sobre as mesmas condições de configuração. A coluna identificada por $Q$ é a quantidade de instâncias e $r$ o raio de cobertura. Todas suas abordagem, exceto a branch and bound utilizaram de técnicas de relaxação lagrangiana baseadas em algumas restrições. Vale ressaltar que essas instâncias, apesar de serem geradas a partir da mesma base de dados, não são idênticas, devido ao fato como são geradas. O gap fornecido pelo autor é reportado pelo resolvedor utilizado em seu trabalho, o CPLEX.

Tabela 3. Instâncias de mesmo tamanho de Marianov et al. (2015)

\begin{tabular}{|c|c|c|c|c|c|c|c|c|c|c|c|}
\hline \multirow[b]{2}{*}{$\mathbf{Q}$} & \multirow[b]{2}{*}{$\mathbf{r}$} & \multirow[b]{2}{*}{$|\mathbf{V}|$} & \multirow[b]{2}{*}{$|\mathbf{A}|$} & \multicolumn{2}{|c|}{ Branch and Bound } & \multicolumn{2}{|c|}{$\begin{array}{l}\text { Relaxação das } \\
\text { restrições } \\
\text { f }>=0 \text { e } x>=0\end{array}$} & \multicolumn{2}{|c|}{$\begin{array}{l}\text { Relaxação lagrangiana da } \\
\text { restrição de equilíbrio de } \\
\text { fluxo }\end{array}$} & \multicolumn{2}{|c|}{$\begin{array}{l}\text { Relaxação lagrangiana das restrições } \\
\text { de cobertura e colocação de cabos nas } \\
\text { trincheiras }\end{array}$} \\
\hline & & & & $\mathbf{T}(\mathbf{s})$ & Gap & $\mathbf{T}(\mathbf{s})$ & Gap & $\mathbf{T}(\mathrm{s})$ & Gap & $\mathbf{T}(\mathbf{s})$ & Gap \\
\hline 5 & 6,7 e 8 & 200 & 800 & 33258,67 & $9,97 \%$ & 33228,73 & $8,03 \%$ & 241,20 & $11,90 \%$ & 1371,07 & $8,78 \%$ \\
\hline
\end{tabular}

Nota-se que o resolvedor GUROBI encontrou o ótimo para todas instâncias e em tempo estritamente menor que as outras abordagens comparadas. As Abordagens heurísticas não chegaram a atingir o ótimo, porém se aproximam muito, principalmente nas instâncias de 100 vértices. Com o intuito de entender o motivo por não terem atingido o ótimo, foi realizada uma análise de algumas soluções e notado que existe uma grande dificuldade do algoritmo encontrar os servidores primários e alocar adequadamente os servidores secundários, 
o que é de se esperar para um problema combinatorial como o pCTP. É notável também a baixa média de gap do VNS, mesmo para instâncias maiores de 200 vértices, diferente do BRKGA.

Nota-se também pelas tabelas, que o gap encontrado pelas abordagens propostas se aproximam e chegam a superar alguns resultados apresentados por Marianov et al. (2015), destacando-se o resolvedor GUROBI, com soluções ótimas e tempo de execução estritamente menor.

\section{CONSIDERAÇÕES FINAIS}

Este artigo propôs duas abordagem heurísticas, uma delas baseada no Biased random-key genetic algorithm e outra no Variable neighborhood search, ambas para o p-cable trench problem with covering, recentemente proposto e ainda pouco explorado. Também foi aplicada uma abordagem exata, que consistiu em utilizar a formulação de programação linear inteira, adaptada dos autores Marianov et al. (2015) e Calik et al. (2016), junto a um resolvedor comercial GUROBI.

Dos experimentos computacionais realizados, o GUROBI demonstrou-se superior às demais abordagens comparadas. A formulação adaptada de forma mais compacta aplicada ao resolvedor pode ter facilitado sua solução, explicando seus bons resultados. Porém devido ao alto custo computacional exigido pelo resolvedor, pressupõe-se que para instâncias de grande porte, o GUROBI tenha problemas ligados a quantidade de memória e processamento que serão exigidos devido a natureza dos algoritmos de programação linear inteira. Por isso as abordagens heurísticas como as apresentadas são ótimas alternativas.

As abordagens heurísticas desenvolvidas apresentaram resultados satisfatórios, porém o VNS apresentou uma certa vantagem por ter apresentado um gap baixo tanto para instâncias de 100 quanto para 200 vértices, diferente do BRKGA que aumentou muito o gap de suas soluções para instâncias de 200 vértices. Vale ressaltar que o tempo de execução do VNS foi melhor, em uma implementação muito mais simples.

Em trabalhos futuros envolvendo o BRKGA, podem ser analisados uma taxa de mutação mais elevada ou a adição de operadores de extermínio para reinicialização da população, assim como novas formas de codificação e decodificação da solução. De forma análoga, pode ser feito para o VNS, modificando sua estrutura algorítmica, realizando buscas locais mais eficientes. Pode-se também trabalhar com outras variantes do pCTPC, como o Capacitated p-Cable Trench Problem with Covering que possui um limite de conexão para cada servidor primário.

\section{REFERÊNCIAS}

Beasley, J. E. (1990), ‘Or-library’, http://people.brunel.ac.uk/ mastjjb/jeb/info.html. 23-052020.

Calik, H., Leitner, M. \& Luipersbeck, M. (2016), 'A benders decomposition based framework for solving cable trench problems', Computers \& Operations Research 81.

Goldbarg, M. (2016), Otimização Combinatória e Meta-Heurísticas: Algoritmos e Aplicações.

Gonçalves, J. \& Resende, M. (2011), 'Biased random-key genetic algorithms for combinatorial optimization', J. Heuristics $17,487-525$.

Gurobi Optimization, L. (2020), 'Gurobi optimizer reference manual'. Acessado: 20-05-20. URL: http://www.gurobi.com

Halim, S. \& Halim, F. (2013), Competitive Programming 3: The New Lower Bound of Programming Contests: Handbook for ACM ICPC and IOI Contestants, Lulu.com. URL: https://books.google.com.br/books? id=tjKYoAEACAAJ

Helsgaun, K. (2009), 'General k-opt submoves for the lin-kernighan tsp heuristic', Mathematical Programming Computation 1, 119-163.

Jeng, D. J.-F., Kim, I. \& Watada, J. (2007), 'Bio-inspired evolutionary method for cable trench pro-blem', International Journal of Innovative Computing Information and Control3, 111-118.

Marianov, V., Gutiérrez-Jarpa, G. \& Obreque, C. (2015), 'P-cable trench problem with covering', XXII EURO working group on locational analysis meeting pp. 75-76.

Marianov, V., Gutiérrez-Jarpa, G., Obreque, C. \& Cornejo, O. (2012), 'Lagrangean relaxation heuristics for the p-cable-trench problem', Computers \& OR39, 620-628.

Meindl, B. \&Templ, M. (2013), 'Analysis of commercial free and open source solvers for linear optimization problems'.

Mladenovic, N. (2003), 'A tutorial on variable neighborhood search'.

Souza, A. S., Pécora, J. E., Loch, G. V. \& Fressato, A. A. (2017), Performance de dois solvers naresolução da metaheurística fix and optimize aplicado ao problema de high school timetabling.

Vasko, F. J., Barbieri, R. S., Rieksts, B. Q., Reitmeyer, K. L. \& Stott, K. L. (2002), 'The cable trench problem: combining the shortest path and minimum spanning tree problems', Computers \& Operations Research 29(5), 441 - 458.

Vasko, F., Landquist, E., Kresge, G., Tal, A., Jiang, Y. \& Papademetris, X. (2015), 'A simple and efficient strategy for solving very large-scale generalized cable-trench problems', Networks. 